\title{
THE COINCIDENCE OF PURE LIPOMA, LEIOMYOMA, AND ENDOMETRIAL CANCER. A CASE REPORT OF THE UTERINE TUMOR TRIPLICITY
}

\author{
Tatána Rešlová ${ }^{1}$, Milan Rešl ${ }^{2}$
}

Charles University in Prague, Faculty of Medicine in Hradec Králové: Department of Obstetrics and Gynecology ${ }^{1}$, The Fingerland Department of Pathology ${ }^{2}$

Summary: A different tumor concurrence of the uterine body is usually encountered as a coincidence of leiomyoma and endometrial cancer. Pure lipomatous tumors or fatty change in the leiomyoma or endometrium are rare affections with some diagnostic peculiarities. We present a case of association of the pure uterine lipoma, leiomyoma, and endometrial cancer as a tumor triplicity, which may contribute, to the pathogenesis of mesenchymal tumors in this location.

Key words: Uterus; Lipoma, Adenocarcinoma; Leiomyoma

\section{Case report}

A 71-year-old obese and hypertonic woman was admitted at hospital with uterine bleeding. Widened endometrium of the myomatously deformed uterus was revealed sonographically. Endometrial cancer confirmed histologically from the curettage was treated by hysterectomy and bilateral adnexectomy without nodal dissection. Radiotherapy was applied in the usual fashion.

Grossly, enlarged and deformed uterus with smooth serosal surface measured 90x60x50 mm (105 g). On section, there were three neoplastic processes situated in the uterine corpus. Its cavity was filled by fragile grayish carcinomatous masses invading the outer half of myometrium (see Figure 1). Second tumor was situated intramurally, well defined (diameter $35 \mathrm{~mm}$ ), and bright yellow colored in all crossections (see Figure). No connection to the endometrium or peritoneum was found. Third affection was of typical myomatous appearance (not shown), measured $15 \mathrm{~mm}$ in diameter, and located inside the myometrium.

Microscopically, the endometrial cancer was typed as a middle differentiated adenocarcinoma of the endometrioid type (T1c; IC). Lipoma feature was quite typical, without admixture of myomatous cells in all examined levels. The third tumor was a leiomyoma with typical immunoreactivity.

Fig. 1: Enlarged uterus containing multiple foci of the endometrial cancer (ca) invading outer half of the myometrium. The well defined pure lipoma (li) situated within the myometrium has a typical brith yellow cut surface. Leiomymoma is not shown at this cut level.

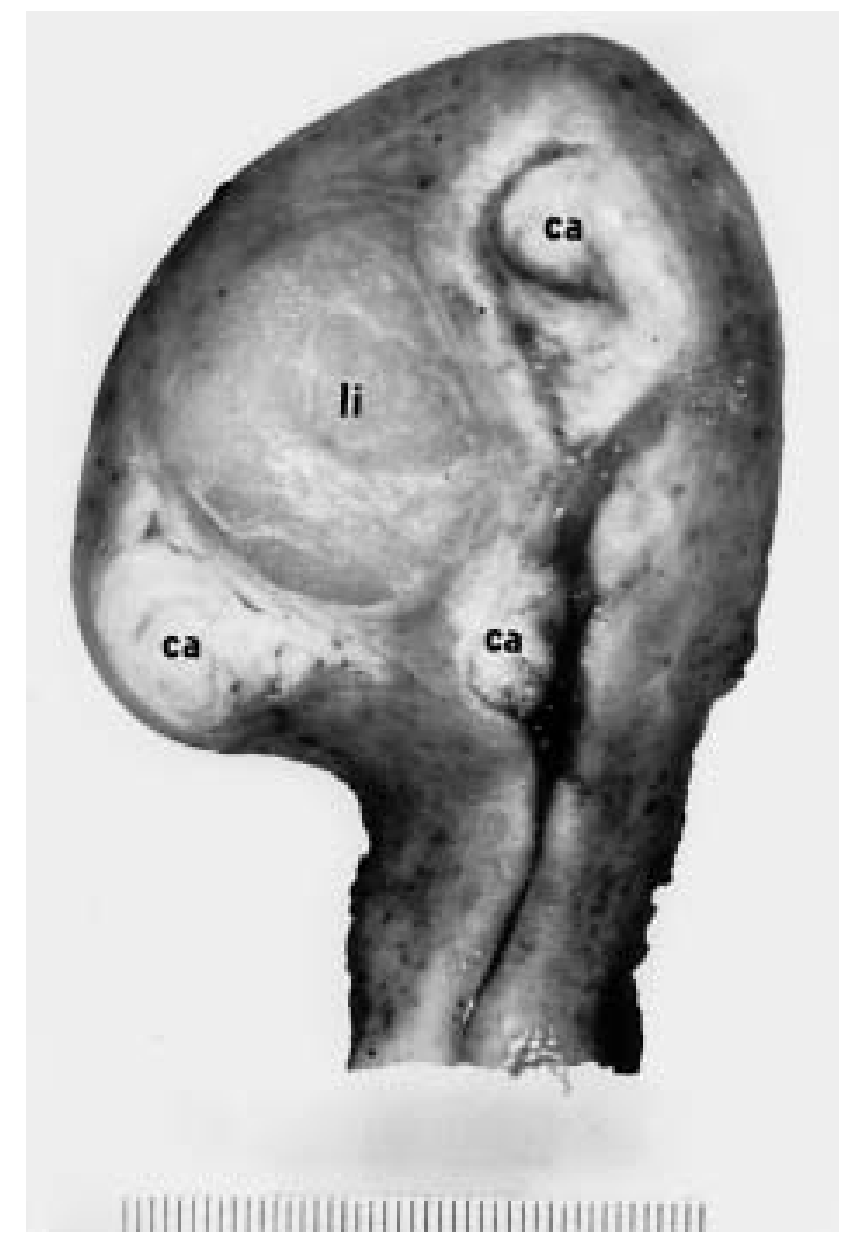


On the basis of these findings the diagnosis of uterine neoplastic triplicity, i.e., endometrioid carcinoma, pure lipoma, and leiomyoma was made.

\section{Discussion}

Presence of adipose tissue in the curettage is an exceptional situation leading to the prompt ultrasonographic control of low abdominal segment. Possibility of the uterine wall perforation and sampling error with mesenterial fat admixture is highly suspected. All other findings of fatty tissue in the curettage may be signs of rare affections arising either in the endometrial or myometrial zones.

Pure endometrial fatty change demonstrates a stromal cell potential for differentiation into adipose phenotype probably induced by inflammation in the neighboring tissue. This process may develop during short time, which is supported by presence of adipocytes within the functional endometrial layer (5). Its significance is not completely understood.

Myometrial fatty tumors are more often reported as lipoleiomyomas. Their incidence was ascertained to be about $0.8 \%$ in the extensively examined leiomyomas (1). Pathogenically, adipose component of the lipoleiomyomas probably represent a metaplastic process within a leiomyoma. This theory is supported by perivascular location of immature cells differentiating into adipocytes immunoreactive with smooth muscle markers (6). Ischemic changes within the leiomyoma may be a possible inducing factor for this change.

A pure uterine lipoma is a very rare affection appears to be of heterogeneous nature. We hypothesize that lipoma or leiomyoma should represent two endpoints of separate differentiation pathways from the primitive mesenchymal (mullerian) progenitor cell. Our case should demonstrate an exceptional situation in which both differentiation path- ways, i.e., into the leiomyoma and lipoma were finished coincidentally. Moreover, literature data suggest that stromal cell also develops from the same progenitor cell (4).

We were not able to find in the English language literature the tumor coincidence of described type. Di Gesu et al. (2) and Douvier et al. (3) reported the association between uterine lipoma and endometrial cancer as a tumor duplicity. These cases were postmenopausal women presented with vaginal bleeding and treated by hysterectomy without lymph node dissection like in our case.

\section{References}

1. Dellacha A, Di Marco A, Foglia G, Fulcheri E. Lipoleiomyoma of the uterus. Pathologica 1997;89:737-41.

2. Di Gesu G, Cormio G, Di Vagno G, Melilli GA, Renzulli G, Selvaggi L. Pure lipoma of the uterus in association with endometrial carcinoma. Eur J Obstet Gynecol Reprod Biol 1998;80:199-200.

3. Douvier S, Nabholtz JM, Brechet E, Collin F, Cuisenier J. The association of uterine lipoma and cancer of the endometrium. Diagnostic and etiopathogenic approach. J Gynecol Obstet Biol Reprod (Paris) 1990;19:301-5.

4. Farhood AI, Abrams J. Immunohistochemistry of endometrial stromal sarcoma. Hum Pathol 1991;22:224-30.

5. Nogales FF, Pavcovich M, Medina MT, Palomino M. Fatty change in the endometrium. Histopathology 1992;20:362-3.

6. Resta L, Maiorano E, Piscitelli D, Botticela MA. Lipomatous tumors of the uterus. Clinico-pathological features of 10 cases with immunocytochemical study of histogenesis. Pathol Res Pract 1994;190:378-83.

Submitted April 2003.

Accepted May 2003.

Doc. MUDr. Tatána Rešlová, Ph.D., Charles University in Prague, Faculty of Medicine in Hradec Králové, Department of Obstetrics and Gynecology, 50005 Hradec Králové, Czech Republic. e-mail: reslova@fnhk.cz 